\title{
Kinetic simulation of nonequilibrium Kelvin-Helmholtz instability
}

\author{
Chuandong Lin, ${ }^{1, *}$ Kai H. Luo, ${ }^{1,2, \dagger}$ Yanbiao Gan, ${ }^{3}$ and Zhipeng Liu ${ }^{4}$ \\ ${ }^{1}$ Center for Combustion Energy; Key Laboratory for Thermal \\ Science and Power Engineering of Ministry of Education, \\ Department of Energy and Power Engineering, \\ Tsinghua University, Beijing 100084, China. \\ ${ }^{2}$ Department of Mechanical Engineering, University College London, \\ Torrington Place, London WC1E 7JE, UK \\ ${ }^{3}$ North China Institute of Aerospace Engineering, Langfang 065000, China \\ ${ }^{4}$ Department of physics, school of science, \\ Tianjin Chengjian University, Tianjin, 300384
}

(Dated: September 19, 2018) 


\begin{abstract}
The recently developed discrete Boltzmann method (DBM), which is based on a uniform linear evolution equation and has high parallel efficiency, is employed to investigate the dynamic nonequilibrium process of Kelvin-Helmholtz instability (KHI). It is found that, the relaxation time always strengthens the global nonequilibrium (GNE), entropy of mixing, and free enthalpy of mixing. Specifically, as a combined effect of physical gradients and nonequilibrium area, the GNE intensity first increases but decreases during the whole life-cycle of KHI. The growth rate of entropy of mixing shows firstly reducing, then increasing, and finally decreasing trends during the KHI process. While the free enthalpy of mixing is opposite to the entropy of mixing. Detailed explanations are as below. (i) Initially, binary diffusion smooths quickly the sharp gradient in the mole fraction, which results in a steeply decreasing mixing speed. (ii) Afterwards, the mixing process is significantly promoted by the increasing length of material interface in the evolution of the KHI. (iii) As physical gradients are smoothed due to the binary diffusion and dissipation, the mixing speed reduces and approaches zero in the final stage. Moreover, with the increasing Atwood number, the global strength of viscous stresses on the heavy (light) medium reduces (increases), because the heavy (light) medium has a relatively small (large) velocity change. Furthermore, for a larger Atwood number, the peaks of nonequilibrium manifestations emerge earlier, the entropy of mixing and free enthalpy of mixing change faster, because the KHI initiates a higher growth rate.
\end{abstract}

PACS numbers: 47.20.Ft; 47.11.-j; 51.10.+y

\footnotetext{
* chuandonglin@163.com

† K.Luo@ucl.ac.uk
} 


\section{INTRODUCTION}

The Kelvin-Helmholtz instability (KHI), named after Lord Kelvin and Hermann von Helmholtz, arises at the perturbed interface between fluids in shear motion, and leads to the formation of vortices and turbulence $[1,2]$. KHI phenomena are ubiquitous in nature and engineering [3-5]. Examples include waves on a windblown ocean or sand dune, swirling cloud billows, the stream structure of solar corona, the helical wave motion in ionized comet tails, the Great Red Spot in Jupiters atmosphere, the Eagle Nebula in astrophysics, the reacting mixing layers in combustion, and the ignition in inertial confinement fusion. The $\mathrm{KHI}$ is one of the most fundamental and famous instabilities in fluid dynamics, and it is often coupled with other instabilities, such as Rayleigh-Taylor Instability [6, 7], RichtmyerMeshkov instability [8,9], etc. Hence, due to its great importance, the KHI has been studied extensively in experimental $[7,10,11]$, theoretical [6], and numerical fields $[12,13]$.

Particularly, with the rapidly improving computational facilities and algorithms, numerical simulation has achieved more and more successes in scientific research and engineering application. Roughly speaking, there are three kinds of numerical methods: microscopic $[14,15]$, mesoscopic $[13,16]$, and macroscopic approaches [17], respectively. Microscopic models, such as molecular dynamics [14] and direct simulation Monte Carlo [15], describe the location and motion of particles in detail, but usually demand too high computational cost. Macroscopic schemes, such as direct numerical simulation, large eddy simulation, and Reynolds-averaged Navier-Stokes (NS) equations, improve computational efficiency significantly, but lose various thermodynamic nonequilibrium information which plays an essential role in interfacial dynamics. To reach a compromise between the computational cost and physical fidelity, a promising method is mesoscopic kinetic modelling based on the Boltzmann equation.

In the last three decades, the kinetic-bsed lattice Boltzmann method (LBM) [18-20] has been developed and become a powerful tool for investigating coherent structures and detailed nonlinear dynamics of complex flows, including the KHI [21-26]. However, most researchers were more likely to take the LBM as a numerical solver of hydrodynamic equations rather than a kinetic model of nonequilibrium systems. Therefore, little consideration has been given to the kinetic nature of related systems. As a novel variant of the LBM, the discrete Boltzmann method (DBM) overcomes the above issue [27-32]. It has the capability of 
simulating the dynamic process of KHI properly by incorporating essential nonequilibrium effects [27-32]. In 2011, Gan et al. proposed a kinetic model for compressible systems and studied the KHI under the influence of velocity and density gradients [27]. This model recovers the Euler equations in the continuum limit, and works for single-component flows. In 2016, Lin et al. developed a DBM for two-component systems [31]. This model recovers the compressible NS equations when the Knudsen number approaches zero [31], and provides more accurate physical information than the former [27]. In the current work, we employ the two-component DBM to study the nonequilibrium process of KHI.

This paper is organized as follows: in Sec. II, we introduce the DBM that provides detailed nonequilibrium information and high parallel efficiency. In Sec. III, we use the DBM to study the nonequilibrium effects, entropy of mixing, and free enthalpy of mixing in the KHI process. Finally, conclusions are given in Sec. IV.

\section{DISCRETE BOLTZMANN MODEL}

In this work, simulation is based on two-dimensional description, with $D=2$ representing the space dimension. The discrete Boltzmann equation with single collision term takes the form,

$$
\frac{\partial f_{k i}^{\sigma}}{\partial t}+\mathbf{v}_{k i} \cdot \nabla f_{k i}^{\sigma}=-\frac{1}{\tau^{\sigma}}\left(f_{k i}^{\sigma}-f_{k i}^{\sigma e q}\right),
$$

where $t$ indicates the time, $\mathbf{v}_{k i}$ the discrete velocity, $\sigma$ the fluid species, $f_{k i}^{\sigma}\left(f_{k i}^{\sigma e q}\right)$ the discrete (equilibrium) distribution function, and $\tau^{\sigma}$ the relaxation time. Note that, for a twocomponent system $(\sigma=A$ and $B$ ), the relaxation times of the two species should be equal in order to ensure the local momentum conservation, i.e., $\tau^{A}=\tau^{B}=\tau$ [33]. The relaxation time is usually a function of species concentration. For simplicity, it is given a constant here. Additionally, we adopt the second Runge-Kutta scheme for the time derivative and the second order nonoscillatory and nonfree-parameter dissipative finite difference scheme [34] for the space derivatives in Eq. (1).

The discrete velocity reads, $\mathbf{v}_{k i}=v_{k i x} \mathbf{e}_{x}+v_{k i y} \mathbf{e}_{y}$, with $v_{k i x}=v_{k} \cos (2 \pi i / 8)$, $v_{k i y}=$ $v_{k} \sin (2 \pi i / 8)$. The suffix $i$ indicates the direction of the discrete velocity, and $k$ indicates the $k$-th group of the particle velocities whose speed is $v_{k}$. To be specific, $i=1,2, \ldots, 8$ and $k=0,1, \ldots, 4$. To ensure the simulation robustness, an optimal choice is $0=v_{0}<v_{1}<$ $v_{2}<v_{3}<v_{4}$. Figure 1 delineates the sketch of the discrete-velocity-model [35]. 


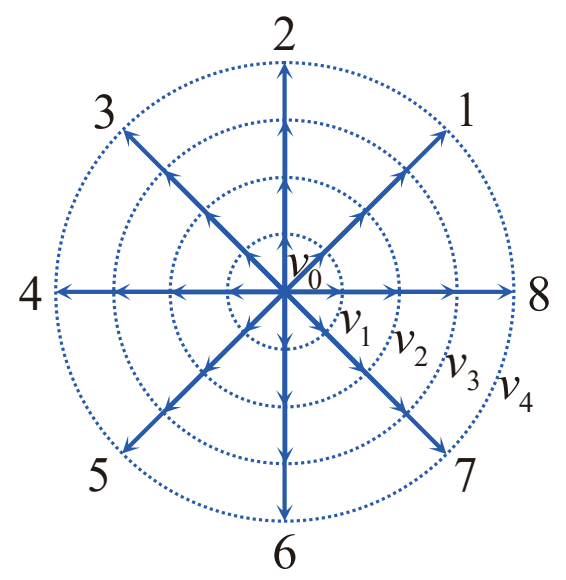

FIG. 1. Sketch of the discrete-velocity-model.

The expression of $f_{k i}^{\sigma e q}$ reads,

$$
\begin{aligned}
f_{k i}^{\sigma e q}= & n^{\sigma} F_{k}\left[\left(1-\frac{u^{2}}{2 \theta^{\sigma}}+\frac{u^{4}}{8 \theta^{\sigma 2}}\right)+\frac{v_{k i \varepsilon} u_{\varepsilon}}{\theta^{\sigma}}\left(1-\frac{u^{2}}{2 \theta^{\sigma}}\right)\right. \\
& +\frac{v_{k i \varepsilon} v_{k i \pi} u_{\varepsilon} u_{\pi}}{2 \theta^{\sigma 2}}\left(1-\frac{u^{2}}{2 \theta^{\sigma}}\right)+\frac{v_{k i \varepsilon} v_{k i \pi} v_{k i \vartheta} u_{\varepsilon} u_{\pi} u_{\vartheta}}{6 \theta^{\sigma 3}} \\
& \left.+\frac{v_{k i \varepsilon} v_{k i \pi} v_{k i \vartheta} v_{k i \xi} u_{\varepsilon} u_{\pi} u_{\vartheta} u_{\xi}}{24 \theta^{\sigma 4}}\right]
\end{aligned}
$$

with the flow speed $u$, and $\theta^{\sigma}=T / m^{\sigma}$, where $m^{\sigma}$ represents the molar mass, $T$ the temperature. The weighting coefficients are,

$$
\begin{aligned}
F_{k}= & {\left[v_{k}^{2}\left(v_{k}^{2}-v_{k+1}^{2}\right)\left(v_{k}^{2}-v_{k+2}^{2}\right)\left(v_{k}^{2}-v_{k+3}^{2}\right)\right]^{-1} } \\
& \times\left[48 \theta^{\sigma 4}-6\left(v_{k+1}^{2}+v_{k+2}^{2}+v_{k+3}^{2}\right) \theta^{\sigma 3}\right. \\
& +\left(v_{k+1}^{2} v_{k+2}^{2}+v_{k+2}^{2} v_{k+3}^{2}+v_{k+3}^{2} v_{k+1}^{2}\right) \theta^{\sigma 2} \\
& \left.-\frac{1}{4} v_{k+1}^{2} v_{k+2}^{2} v_{k+3}^{2} \theta^{\sigma}\right], \\
F_{0}= & 1-8\left(F_{1}+F_{2}+F_{3}+F_{4}\right),
\end{aligned}
$$

where the suffixes

$$
\bmod \{k+l, 4\}=\left\{\begin{array}{ccc}
k+l & \text { for } & k+l \leq 4 \\
k+l-4 & \text { for } & k+l>4
\end{array}\right.
$$

The mole concentration, mass density, flow velocity, and energy of species $\sigma$ are calculated as below,

$$
n^{\sigma}=\sum_{k i} f_{k i}^{\sigma}
$$




$$
\begin{gathered}
\rho^{\sigma}=m^{\sigma} n^{\sigma} \\
\mathbf{u}^{\sigma}=\frac{1}{n^{\sigma}} \sum_{k i} f_{k i}^{\sigma} \mathbf{v}_{k i}, \\
E^{\sigma}=\frac{1}{2} m^{\sigma} \sum_{k i} f_{k i}^{\sigma} \mathbf{v}_{k i} \cdot \mathbf{v}_{k i} .
\end{gathered}
$$

The mole concentration, mass density, flow velocity and temperature of the mixing are $n=\sum_{\sigma} n^{\sigma}, \rho=\sum_{\sigma} \rho^{\sigma}, \mathbf{u}=\sum_{\sigma}\left(\rho^{\sigma} \mathbf{u}^{\sigma}\right) / \rho$, and $T=\left(2 \sum_{\sigma} E^{\sigma}-\rho \mathbf{u} \cdot \mathbf{u}\right) / D$, respectively. Mathematically, the coupling between the two sets of Boltzmann equations (1) is achieved via the flow speed $u=|\mathbf{u}|$ and temperature $T$ which enter the expression of the equilibrium distribution functions in Eq. (2). In this way, the cross-collisions of the species $A$ and $B$ exchange physical information between them.

It is easy to prove that the DBM could recover the NS equations in the hydrodynamic limit [31]. Moreover, the DBM can be employed to measure the following nonequilibrium manifestations,

$$
\begin{gathered}
\boldsymbol{\Delta}_{2}^{\sigma *}=m^{\sigma} \sum_{k i}\left(f_{k i}^{\sigma}-f_{k i}^{\sigma e q}\right) \mathbf{v}_{k i}^{*} \mathbf{v}_{k i}^{*}, \\
\boldsymbol{\Delta}_{3,1}^{\sigma *}=m^{\sigma} \sum_{k i}\left(f_{k i}^{\sigma}-f_{k i}^{\sigma e q}\right)\left(\mathbf{v}_{k i}^{*} \cdot \mathbf{v}_{k i}^{*}\right) \mathbf{v}_{k i}^{*}, \\
\boldsymbol{\Delta}_{3}^{\sigma *}=m^{\sigma} \sum_{k i}\left(f_{k i}^{\sigma}-f_{k i}^{\sigma e q}\right) \mathbf{v}_{k i}^{*} \mathbf{v}_{k i}^{*} \mathbf{v}_{k i}^{*}, \\
\boldsymbol{\Delta}_{4,2}^{\sigma *}=m^{\sigma} \sum_{k i}\left(f_{k i}^{\sigma}-f_{k i}^{\sigma e q}\right)\left(\mathbf{v}_{k i}^{*} \cdot \mathbf{v}_{k i}^{*}\right) \mathbf{v}_{k i}^{*} \mathbf{v}_{k i}^{*},
\end{gathered}
$$

with the central velocity, $\mathbf{v}_{k i}^{*}=\mathbf{v}_{k i}-\mathbf{u}$. The second-order tensor $\Delta_{2}^{\sigma *}=\Delta_{2, \alpha \beta}^{\sigma *} \mathbf{e}_{\alpha} \mathbf{e}_{\beta}$ corresponds to the viscous stress tensor, with $\mathbf{e}_{\alpha}$ the unit vector in the $\alpha$ direction. The vector $\boldsymbol{\Delta}_{3,1}^{\sigma *}=\Delta_{3,1, \alpha}^{\sigma *} \mathbf{e}_{\alpha}$ is related to the heat flux. $\boldsymbol{\Delta}_{3}^{\sigma *}=\Delta_{3, \alpha \beta \gamma}^{\sigma *} \mathbf{e}_{\alpha} \mathbf{e}_{\beta} \mathbf{e}_{\gamma}$ and $\boldsymbol{\Delta}_{4,2}^{\sigma *}=$ $\Delta_{4,2, \alpha \beta}^{\sigma *} \mathbf{e}_{\alpha} \mathbf{e}_{\beta}$ are higher order nonequilibrium quantities beyond traditional NS models. To roughly estimate the "degree of nonequilibrium", we introduce the following four condensed measures, $\left|\boldsymbol{\Delta}_{2}^{\sigma *}\right|=\sqrt{\Delta_{2, x x}^{\sigma * 2}+2 \Delta_{2, x y}^{\sigma * 2}+\Delta_{2, y y}^{\sigma * 2}},\left|\Delta_{3,1}^{\sigma *}\right|=\sqrt{\Delta_{3,1, x}^{\sigma * 2}+\Delta_{3,1, y}^{\sigma * 2}},\left|\Delta_{3}^{\sigma *}\right|=$ $\sqrt{\Delta_{3, x x x}^{\sigma * 2}+3 \Delta_{3, x x y}^{\sigma * 2}+3 \Delta_{3, x y y}^{\sigma * 2}+\Delta_{3, y y y}^{\sigma * 2}}$, and $\left|\Delta_{4,2}^{\sigma *}\right|=\sqrt{\Delta_{4,2, x x}^{\sigma * 2}+2 \Delta_{4,2, x y}^{\sigma * 2}+\Delta_{4,2, y y}^{\sigma * 2}}$. The value of $\left|\boldsymbol{\Delta}_{2}^{\sigma *}\right|$ is used to measure the strength of the viscous stress; $\boldsymbol{\Delta}_{3,1}^{\sigma *}$ indicate the intensity of the heat flux; $\left|\boldsymbol{\Delta}_{3}^{\sigma *}\right|$ and $\left|\boldsymbol{\Delta}_{4,2}^{\sigma *}\right|$ represent the degrees of $\boldsymbol{\Delta}_{3}^{\sigma *}$ and $\boldsymbol{\Delta}_{4,2}^{\sigma *}$, respectively. Physically, $\left|\boldsymbol{\Delta}_{2}^{\sigma *}\right|,\left|\boldsymbol{\Delta}_{3,1}^{\sigma *}\right|,\left|\boldsymbol{\Delta}_{3}^{\sigma *}\right|$, and $\left|\boldsymbol{\Delta}_{4,2}^{\sigma *}\right|$ are zero only in equilibrium. Otherwise, they are nonzero, and become larger as the fluid system departs further from its local equilibrium. 


\begin{tabular}{ccccccccc}
\hline \hline Tests & Test-1 & Test-2 & Test-3 & Test-4 & Test-5 & Test-6 & Test-7 & Test-8 \\
\hline Processing cores & $1 \times 1$ & $2 \times 2$ & $4 \times 4$ & $8 \times 8$ & $16 \times 16$ & $32 \times 32$ & $64 \times 64$ & $128 \times 128$ \\
Running time (s) & 755840 & 198112 & 55120 & 13792 & 3476 & 881 & 221 & 59 \\
Efficiency & $100 \%$ & $95.4 \%$ & $85.7 \%$ & $85.6 \%$ & $84.9 \%$ & $83.8 \%$ & $83.5 \%$ & $82.0 \%$ \\
\hline \hline
\end{tabular}

TABLE I. Parallel efficiency study of the DBM.

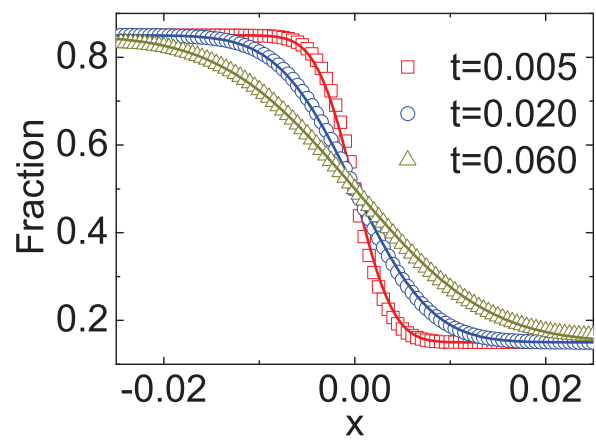

FIG. 2. Mole fractions $Y^{A}$ in the binary diffusion at time instants: $t=0.005,0.20$, and 0.60 , respectively. Symbols stand for DBM simulation results, and solid lines for the corresponding analytical solutions.

Theoretically, similar to the standard LBM, the DBM has the outstanding merits of simplicity for programming and high parallel efficiency, because the discrete Boltzmann equation is uniformly linear and all the information transfer in DBM is local in time and space. However, the parallel efficiency of our DBM has never been demonstrated in a numerical way. To this end, we conduct simulations using parallel programming based on the Message-Passing Interface standard. Calculations are carried out on the supercomputer ARCHER. Table I exhibits the parallel efficiency. Eight tests are performed using the number of processing cores, $N_{p}=1 \times 1,2 \times 2,4 \times 4,8 \times 8,16 \times 16,32 \times 32,64 \times 64$, and $128 \times 128$, respectively. The mesh is chosen as $N_{x} \times N_{y}=3200 \times 3200$. The other parameters are identical to those for Fig. 4. After 8000 iterative steps, the running time is $t_{1}=755840$, $t_{2}=198112, t_{3}=55120, t_{4}=13792, t_{5}=3476, t_{6}=881, t_{6}=221$, and $t_{8}=59$ seconds, respectively. Define the parallel efficiency as $E_{p}=N_{p} t_{i} / t_{1}$, with $i=1,2, \ldots, 6$. Then the efficiency is $100 \%, 95.4 \%, 85.7 \%, 85.6 \%, 84.9 \%, 83.8 \%, 83.5 \%$, and $82.0 \%$ for the eight tests, respectively. It is evident that the parallel efficiency of the DBM is quite high. 
To validate its capability of describing the interaction between two species, this DBM is used to simulate the binary diffusion. Initially, the mole concentration is described by the following step function,

$$
\left\{\begin{array}{c}
\left(n^{A}, n^{B}\right)_{L}=(0.9,0.1) \\
\left(n^{A}, n^{B}\right)_{R}=(0.1,0.9)
\end{array}\right.
$$

where the suffixes $L$ and $R$ indexes the left and right parts, respectively. The temperature is $T=1$, the velocity $\mathbf{u}=0$, the molecular mass $m^{\sigma}=1$, the relaxation time $\tau=10^{-3}$, the temporal step $\Delta t=10^{-5}$, the spatial step $\Delta x=\Delta y=5 \times 10^{-4}$, the mesh grid $N_{x} \times N_{y}=$ $100 \times 1$. Figure 2 displays the mole fraction of species $A$ at three time instants. Symbols are for DBM results, and solid lines for the analytical solution

$$
Y^{\sigma}=\frac{1}{2}+\frac{\Delta Y^{\sigma}}{2} \operatorname{Erf}\left(\frac{x}{\sqrt{4 \chi t}}\right)
$$

where $\Delta Y^{\sigma}=0.7$ is the initial mole fraction difference, and $\chi=10^{-3}$ the diffusion coefficient. We can find a satisfying agreement between the two sets of results.

\section{SIMULATION AND INVESTIGATION}

In this section, let us focus on the KHI. The initial field configuration is delineated in Fig. 3. The left part is occupied by species $A$ with an upward velocity $\mathbf{u}_{L}=u_{L} \mathbf{e}_{y}$ and a mole concentration $n_{L}^{A}$, the right part is full of $B$ with a downward velocity $\mathbf{u}_{R}=-u_{R} \mathbf{e}_{y}$ and a mole concentration $n_{R}^{B}$. Between them is a perturbed interface with a transition layer. Mathematically,

$$
\left\{\begin{array}{l}
n^{\sigma}=\frac{n_{L}^{\sigma}+n_{R}^{\sigma}}{2}-\frac{n_{L}^{\sigma}-n_{R}^{\sigma}}{2} \tanh \left(\frac{x-x_{0}+W \cos (k y)}{D_{\rho}}\right), \\
\mathbf{u}=\frac{\mathbf{u}_{L}+\mathbf{u}_{R}}{2}-\frac{\mathbf{u}_{L}-\mathbf{u}_{R}}{2} \tanh \left(\frac{x-x_{0}+W \cos (k y)}{D_{u}}\right),
\end{array}\right.
$$

where $D_{\rho}\left(D_{u}\right)$ represents the width of density (velocity) transition layer, $x_{0}$ denotes the averaged $x$ position of the cosine-shaped interface, $W$ and $k$ are the perturbation amplitude and wave number, respectively. The pressure $p$ is equal at the two sides of the interface. The two species have an identical velocity and temperature at the same location. The length and width of the physical domain are $L_{x}=1.5$ and $L_{y}=0.5$, respectively. The parameters are chosen as $n_{L}^{A}=1, n_{R}^{A}=0, n_{L}^{B}=0, n_{R}^{B}=1, u_{L}=0.5, u_{R}=0.5, D_{\rho}=D_{u}=L_{x} / 300$, $W=L_{x} / 200, x_{0}=L_{x} / 2, k=2 \pi / L_{y}$. Furthermore, boundary conditions are as follows: the inflow/outflow (zero gradient) boundary conditions in the $x$ direction, the periodic boundary 
conditions in the $y$ direction. In addition, all quantities are expressed in nondimensional forms, i.e., the widely accepted LB units [27], in this work.

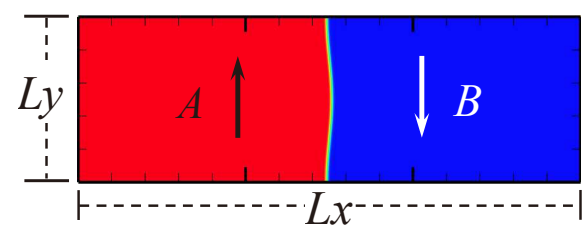

FIG. 3. Field configuration.

\section{A. Grid convergence test}

Grid convergence is one of the most important issues in numerical simulations. To verify the validity of the simulation, a grid convergence test is firstly carried out using various grids: $N_{x} \times N_{y}=750 \times 250,1500 \times 500,3000 \times 1000$, and $6000 \times 2000$, respectively. The time step is $\Delta t=2.5 \times 10^{-5}$, the relaxation time $\tau=2 \times 10^{-4}$, the molar mass $m^{A}=3$, and $m^{B}=1$. Figure 4 delineates the molar fraction $Y^{A}\left(=n^{A} / n\right)$ along the line $y=L_{y} / 2$ at the time $t=1$. As one can see, with increasing mesh grids, the numerical errors become smaller and smaller. The simulation results between grids $6000 \times 2000$ and $3000 \times 1000$ are small enough. Taking account of both accuracy and efficiency, we carry out this simulation on the gird $3000 \times 1000$.

Figure 5 depicts the molar fraction $Y^{A}$, pressure $p(=n T)$, and vorticity $\omega\left(=\partial_{x} u_{y}-\partial_{y} u_{x}\right)$ at time instants $t=0.5,1.0,2.0$, and 3.0, respectively. It is evident that the perturbed interface begins to wiggle due to the velocity shear at $t=0.5$. After the initial linear growth stage, a rolled-up vortex emerges around the interface at $t=1.0$. Then, at $t=2.0$, a larger vortex is observed in the snapshot. At $t=3.0$, the thickening interfacial layer indicates that KHI promotes the mixing and penetrating of the miscible fluid components. Comparing pressure contours with vorticity, we can find that the minimum pressure is located at the vortex center, and the maximum pressure is at the position where two opposite direction flows encounter each other. The flow field varies with space and time. In fact, the pressure gradient offers the centripetal force required by the rotating flows. Moreover, the interface is continuous and smooth, as shown in Fig. 5. The coherent structures demonstrate that the DBM has a good ability of capturing interface deformation. 


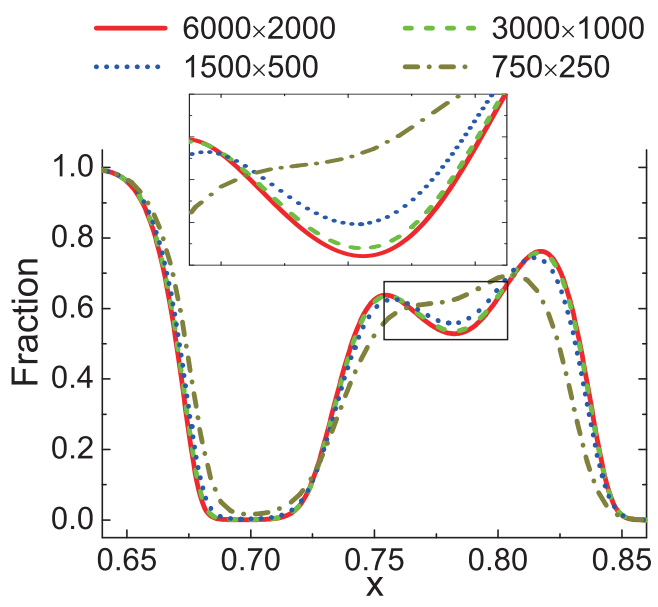

FIG. 4. Grid convergence test: molar fraction of species $A$ along the line $y=L_{y} / 2$ at the time $t=1$. The solid, dashed, dotted, and dot-dashed lines denote the mesh grids $N_{x} \times N_{y}=6000 \times 2000$, $3000 \times 1000,1500 \times 500$, and $750 \times 250$, respectively. The inset enlarges the outlined rectangular region.

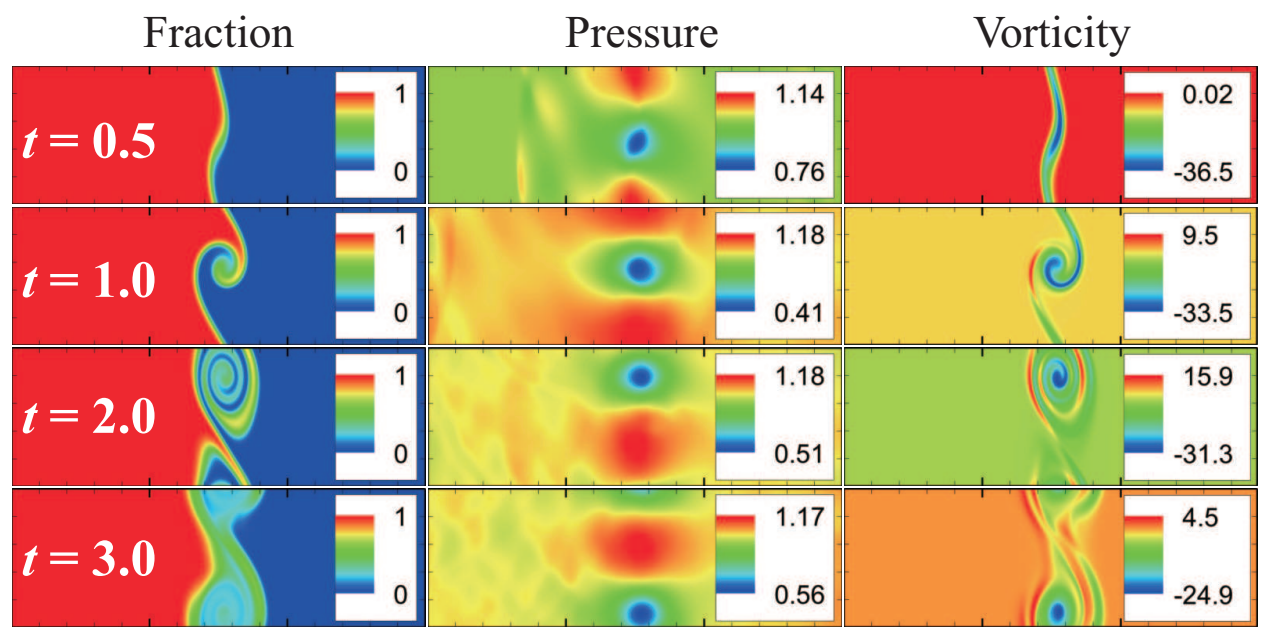

FIG. 5. Contours of physical quantities at time instants $t=0.5,1.0,2.0$, and 3.0, respectively. The leftmost, middle, and rightmost columns show the snapshots of the molar fraction of $A$, pressure, and vorticity, respectively.

\section{B. Nonequilibrium effects}

As an initial application, we preliminarily study two kinds of nonequilibrium effects, $\left|\Delta_{2}^{\sigma *}\right|$ and $\left|\boldsymbol{\Delta}_{3,1}^{\sigma *}\right|$, in the evolution of KHI. To give an intuitive impression, Fig. 6 depicts 


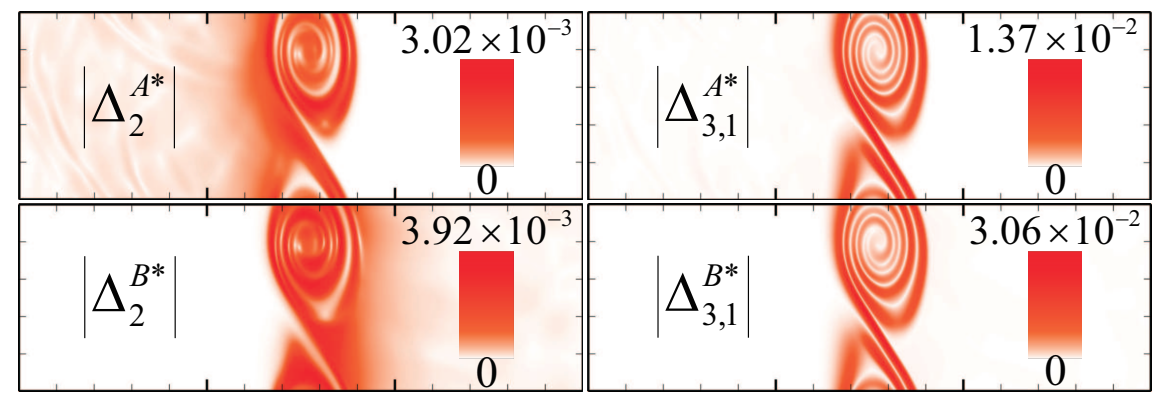

FIG. 6. Contours of nonequilibrium quantities, $\left|\boldsymbol{\Delta}_{2}^{\sigma *}\right|$ and $\left|\boldsymbol{\Delta}_{3,1}^{\sigma *}\right|$, at the moment $t=2.0$.

the contours of them at the moment $t=2.0$. The values of $\left|\boldsymbol{\Delta}_{2}^{A *}\right|$ and $\left|\boldsymbol{\Delta}_{2}^{B *}\right|$ are greater than zero around the vortex where the shear is significant, while they are close to zero far away from the interface where the velocity difference is low. Meanwhile, values of $\left|\boldsymbol{\Delta}_{3,1}^{A *}\right|$ and $\left|\boldsymbol{\Delta}_{3,1}^{B *}\right|$ are large at the contact between the two media, while they approach zero where the interaction between the fluids is weak.
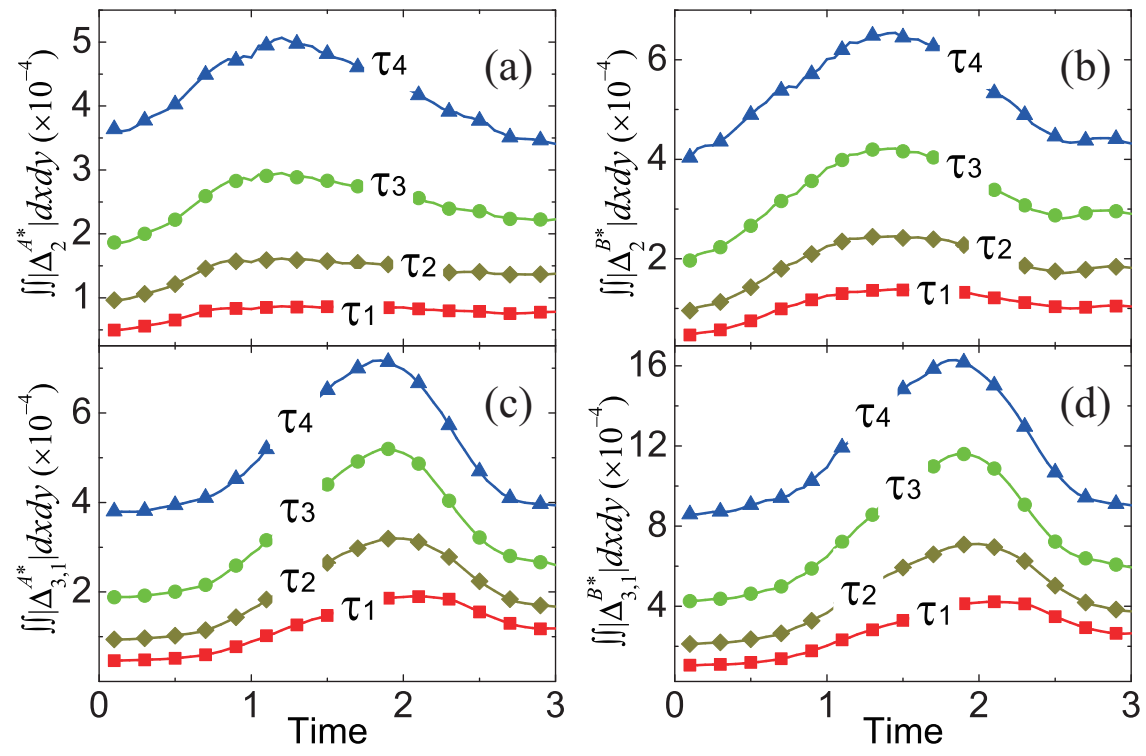

FIG. 7. Evolution of nonequilibrium quantities with various relaxation times: $\tau_{1}=10^{-4}$ (squares), $\tau_{2}=2 \times 10^{-4}$ (diamonds), $\tau_{3}=4 \times 10^{-4}$ (circles), and $\tau_{4}=8 \times 10^{-4}$ (triangles), respectively. (a) $\iint\left|\boldsymbol{\Delta}_{2}^{A *}\right| d x d y$, (b) $\iint\left|\boldsymbol{\Delta}_{2}^{B *}\right| d x d y$, (c) $\iint\left|\boldsymbol{\Delta}_{3,1}^{A *}\right| d x d y$, (d) $\iint\left|\boldsymbol{\Delta}_{3,1}^{B *}\right| d x d y$.

To investigate the influence of relaxation time on the nonequilibrium KHI, we conduct four runs with various relaxation times, $\tau_{1}=10^{-4}, \tau_{2}=2 \times 10^{-4}, \tau_{3}=4 \times 10^{-4}$, and $\tau_{4}=8 \times 10^{-4}$, 
respectively. Let us define the Reynolds number as $R e=\bar{\rho} \bar{u} \bar{L} / \bar{\mu}$, where the characteristic density is $\bar{\rho}=\left(m^{A} n^{A}+m^{B} n^{B}\right) / 2$, the characteristic velocity $\left(u_{L}+u_{R}\right) / 2$, the characteristic length $\bar{L}=L_{y}$, the dynamic viscosity $\bar{\mu}=p \tau$. Correspondingly, $R e=3750,1875,937.5$, and 468.75, for the four runs, respectively. Taking into account of both numerical accuracy and computational efficiency, we chose the spatial step as $\Delta x=\Delta y=2.5 \times 10^{-4}, 5 \times 10^{-4}$, $10^{-3}$, and $2 \times 10^{-3}$, respectively. And the temporal step is $\Delta t=1.25 \times 10^{-5}, 2.5 \times 10^{-5}$, $5 \times 10^{-5}$, and $10^{-4}$, respectively.

Figure 7 plots the evolution of nonequilibrium quantities $\iint\left|\boldsymbol{\Delta}_{2}^{\sigma *}\right| d x d y$ and $\iint\left|\boldsymbol{\Delta}_{3,1}^{\sigma *}\right| d x d y$, respectively, where the integral is extended over all physical space $L_{x} \times L_{y}$. Actually, $\iint\left|\boldsymbol{\Delta}_{2}^{\sigma *}\right| d x d y$ and $\iint\left|\boldsymbol{\Delta}_{3,1}^{\sigma *}\right| d x d y$ indicate the global nonequilibrium effect (GNE) of species $\sigma$ in the physical region from two different points of view. $\iint\left|\boldsymbol{\Delta}_{2}^{\sigma *}\right| d x d y$ stands for the global strength of the viscous stress, while $\iint\left|\Delta_{3,1}^{\sigma *}\right| d x d y$ represents the global intensity of the heat flux. The lines with squares, diamonds, circles, and triangles stand for the relaxation times $\tau_{1}, \tau_{2}, \tau_{3}$, and $\tau_{4}$, respectively. It is evident that the GNEs become stronger for larger relaxation times. The evolution of GNE shows firstly increasing then decreas-

ing trends. $\iint\left|\boldsymbol{\Delta}_{2}^{\sigma *}\right| d x d y$ and $\iint\left|\boldsymbol{\Delta}_{3,1}^{\sigma *}\right| d x d y$ do not reach their peaks simultaneously, and $\iint\left|\boldsymbol{\Delta}_{2}^{\sigma *}\right| d x d y$ reaches the maximum earlier than $\iint\left|\boldsymbol{\Delta}_{3,1}^{\sigma *}\right| d x d y$. Physically, there are competitive mechanisms in the evolution of GNEs. The GNE is associated with the local nonequilibrium effect and the nonequilibrium area. On the one hand, the interface is lengthened with the development of the vortex. The increasing interface enhances the GNEs. On the other hand, the interface is widened in the process of the binary diffusion and thermal conductivity, hence the physical gradients become smooth. The reducing physical gradients weaken the local nonequilibrium effects.

\section{Entropy of mixing}

In thermodynamics, entropy of mixing is part of the increasing entropy when separate systems with different components contact and mix, before the establishment of a thermodynamic equilibrium state. The statistical concept of randomness is utilized for statistical mechanical explanation of the entropy of mixing. Mathematically, the entropy of mixing per unit volume takes the form,

$$
S_{M}=-\sum_{\sigma} n^{\sigma} \ln Y^{\sigma}
$$


where $Y^{\sigma}$ is the molar fraction of species $\sigma$. The entropy of mixing is a typical thermodynamic nonequilibrium variable. It indicates the mixing extent and nonequilibrium process in various miscible fluid systems.

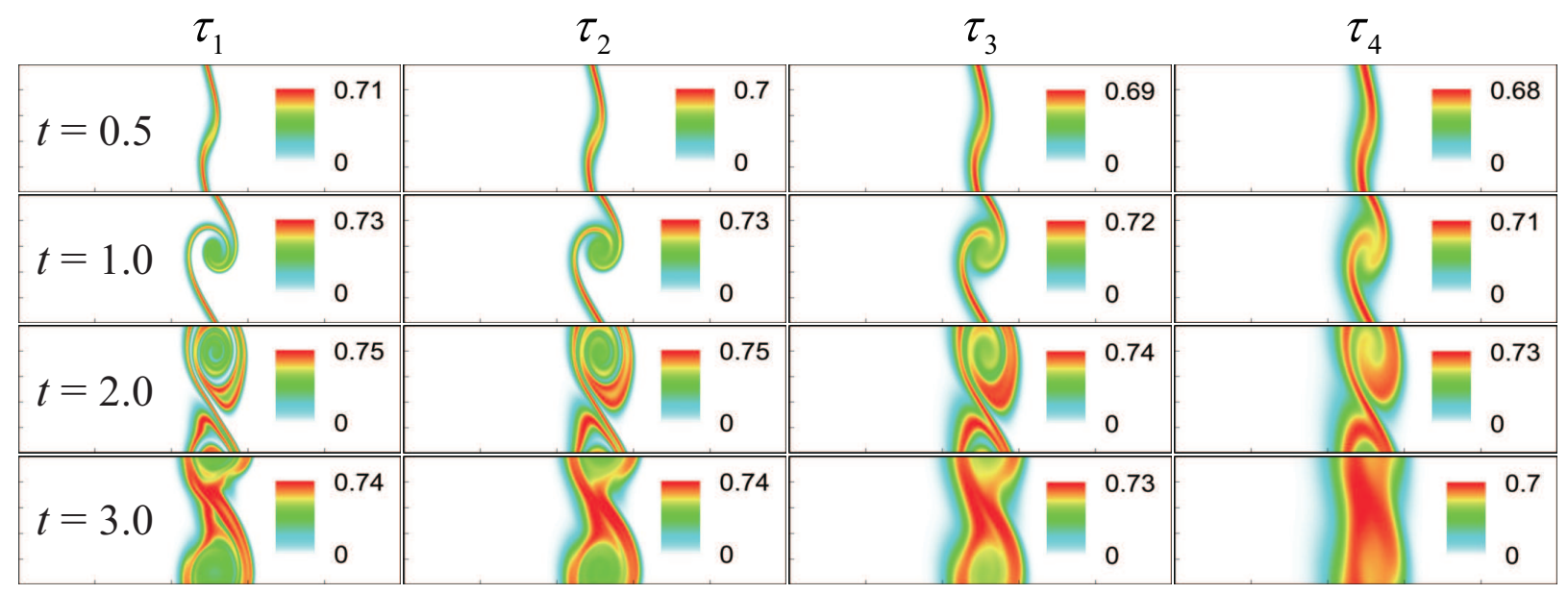

FIG. 8. Contours of the entropy of mixing at time instants $t=0.5,1.0,2.0$, and 3.0, respectively. The four columns, from left to right, correspond to the relaxation times $\tau_{1}=10^{-4}, \tau_{2}=2 \times 10^{-4}$, $\tau_{3}=4 \times 10^{-4}$, and $\tau_{4}=8 \times 10^{-4}$, respectively.

Figure 8 illustrates the snapshots of entropy of mixing in the evolution of KHI. The four columns, from left to right, correspond to cases with relaxation times $\tau_{1}, \tau_{2}, \tau_{3}$, and $\tau_{4}$, respectively. The four rows, from top to bottom, correspond to the time instants $t=0.5$, $1.0,2.0$, and 3.0, respectively. It is clear in each column that the mixing area increases in the evolution of KHI. The rolled-up vortex lengthens the interface and promotes the mixing process. Meanwhile, it can be found in every row that the mixing area is larger for a larger relaxation time, because the diffusion coefficient which enhances the mixing process is proportional to the relaxation time [36].

For the purpose of a quantitative study, we probe the temporal evolution of entropy of mixing with various relaxation times. Figure 9 (a) shows $\iint S_{M} d x d y$, where the integral is extended over all physical region, and (b) plots its growth rate. Obviously, in Fig. 9 (a), the entropy of mixing is higher for a larger relaxation time, which is consistent with Fig. 8. Moreover, it is interesting to see in Fig. 9 (b) that the evolution of its growth rate shows three trends, i.e., firstly reducing, then increasing, and finally decreasing trends. Physically, there are competitive mechanisms in the evolution of the entropy of mixing. (i) 

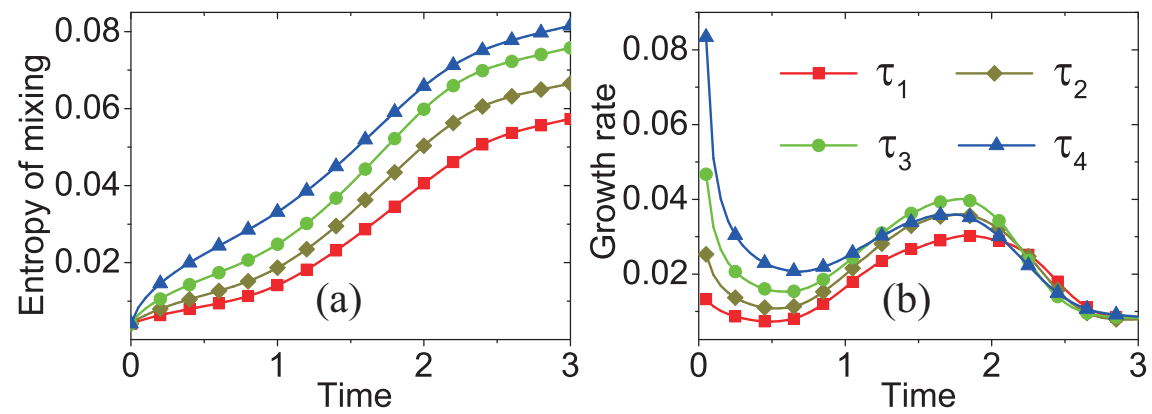

FIG. 9. Evolution of entropy of mixing and its growth rate with various relaxation times: $\tau_{1}=10^{-4}$ (squares), $\tau_{2}=2 \times 10^{-4}$ (diamonds), $\tau_{3}=4 \times 10^{-4}$ (circles), and $\tau_{4}=8 \times 10^{-4}$ (triangles), respectively.

The binary diffusion is fast when the gradient of molar fraction is sharp. The transition layer of the initial interface is relatively narrow, hence the diffusion and mixing is relatively fast. The mixing speed reduces with the increasing width of the transition layer in the early stage. (ii) The mixing process is promoted by the increasing length of material interface with the development of the vortex. In the evolution of KHI, the interface becomes longer and longer, hence the mixing speed increases in the second stage. (iii) With the ongoing diffusion, the material interface becomes wide and the gradients are smoothed. The mixing speed reduces and begins to approach zero, when the gradient becomes smaller and smaller around the vortex, in the final stage. It is of interest to find that the fast diffusion promotes the mixing process directly and initially, but slows the mixing growth indirectly and finally, in the dynamic process of KHI.

\section{Free enthalpy of mixing}

In thermodynamics, free enthalpy of mixing is another interesting thermodynamic nonequilibrium variable. Let us introduce Gibbs free enthalpy of mixing per unit volume as below,

$$
H_{M}=\sum_{\sigma} n^{\sigma} T \ln Y^{\sigma}
$$

Note that $H_{M} \leq 0$ since $0 \leq Y^{\sigma} \leq 1$ and $\ln Y^{\sigma} \leq 0$. In other words, free enthalpy of mixing is always nonpositive, and it is opposite to entropy of mixing that is nonnegative. Mathematically, $H_{M}=-S_{M} T$. 


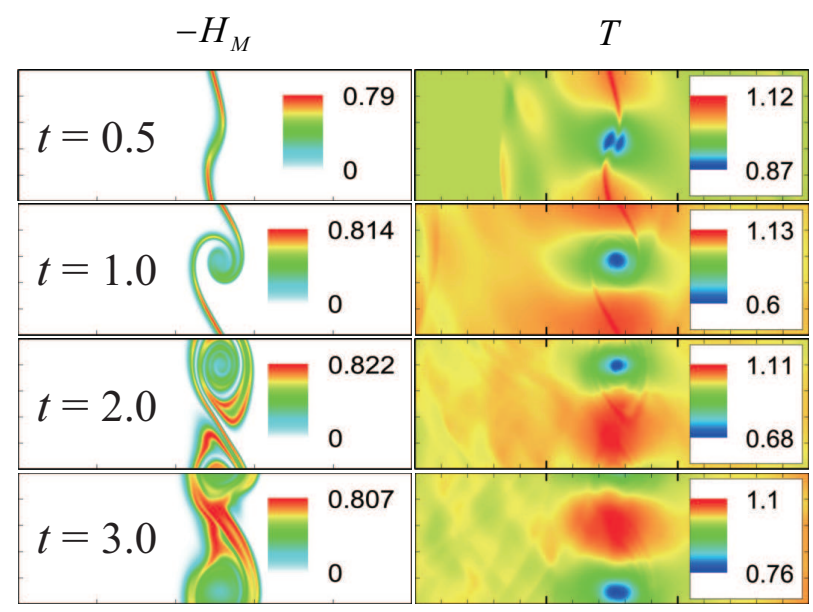

FIG. 10. Contours of minus free enthalpy of mixing $\left(-H_{M}\right)$ and temperature $(\mathrm{T})$ with the relaxation time $\tau=10^{-4}$ at time instants $t=0.5,1.0,2.0$, and 3.0, respectively.

Figure 10 depicts the fields of free enthalpy of mixing and temperature at time instants $t=0.5,1.0,2.0$, and 3.0, respectively. The case corresponds to the first column in Fig. 8 with the relaxation time $\tau_{1}=10^{-4}$. In order to make a clear comparison to the entropy of mixing, the minus free enthalpy of mixing is shown. Since the free enthalpy of mixing is the function of the entropy of mixing and temperature, the contours of temperature are plotted aside. A comparison between Figs. 8 and 10 gives two points. On the one hand, the entropy of mixing and minus free enthalpy of mixing exhibit similar contours, as both of them are located at the interface between the two species. On the other hand, the entropy of mixing and minus free enthalpy of mixing show some different values due to the spatio-temporal variation of temperature in the evolution of KHI.

Figure 11 (a) plots $\iint H_{M} d x d y$, where the integral is extended over all physical region, and (b) illustrates its growth rate. In contrast to the entropy of mixing, the free enthalpy of mixing is lower for a larger relaxation time, and its growth rate displays three different trends, i.e., firstly increasing, then decreasing, and finally increasing trends. The tendency of the growth rate of free enthalpy of mixing is opposite to that of entropy of mixing. The physical mechanisms for the free enthalpy of mixing are identical with that for the entropy of mixing. 

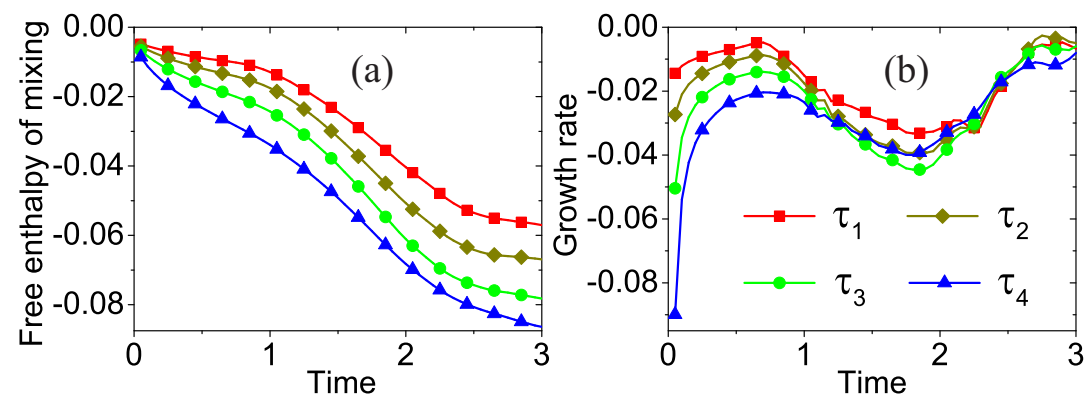

FIG. 11. Evolution of the free enthalpy of mixing and its growth rate with various relaxation times: $\tau_{1}=10^{-4}$ (squares), $\tau_{2}=2 \times 10^{-4}$ (diamonds), $\tau_{3}=4 \times 10^{-4}$ (circles), and $\tau_{4}=8 \times 10^{-4}$ (triangles), respectively.

\section{E. Atwood number}

Atwood number is an important parameter that affects the growth of KH instability. It is defined as

$$
A t=\frac{\rho^{A}-\rho^{B}}{\rho^{A}+\rho^{B}}=\frac{m^{A}-m^{B}}{m^{A}+m^{B}},
$$

with $\rho^{\sigma}=n^{\sigma} m^{\sigma}$ and $n^{\sigma}=1$ in this section. Let us consider four cases, i.e., $m^{A}=1,2,3$, and 4 , with fixed $m^{B}=1$. The corresponding Atwood numbers are $A t_{1}=0, A t_{2}=1 / 3$, $A t_{3}=1 / 2$, and $A t_{4}=3 / 5$, respectively. The other parameters are the same as those in Fig. 5 .

Figure 12 depicts the snapshots of the molar fraction $Y^{A}$, the speed $|\mathbf{u}|\left(=\sqrt{u_{x}^{2}+u_{y}^{2}}\right)$, and the temperature $T$ at time $t=1$ with various Atwood numbers. We can find an upward movement in the vortex center with the increasing Atwood numbers. Physically, the average flow velocity in the $y$ direction, $u_{y}$, is zero around the interface for the case $m^{A}=m^{B}$, and it increases with the increasing mass ratio of species $A$ to $B$, i.e., $R_{m}=m^{A}: m^{B}$. Hence the vortex goes up as the flow speed $u_{y}$ increases with the increasing Atwood numbers. Moreover, it is clear in the first column that the vortex becomes wider in the $x$ direction for a smaller Atwood number. The second column shows that the maximum flow speed increases with the increasing Atwood number. It can be obtained from the first and second columns that the light medium $B$ has larger speed than the heavy one $A$ around the vortex. Finally, the third column shows that, with the increasing Atwood number, the maximum temperature increases and the minimum temperature reduces. It is seen from the first 


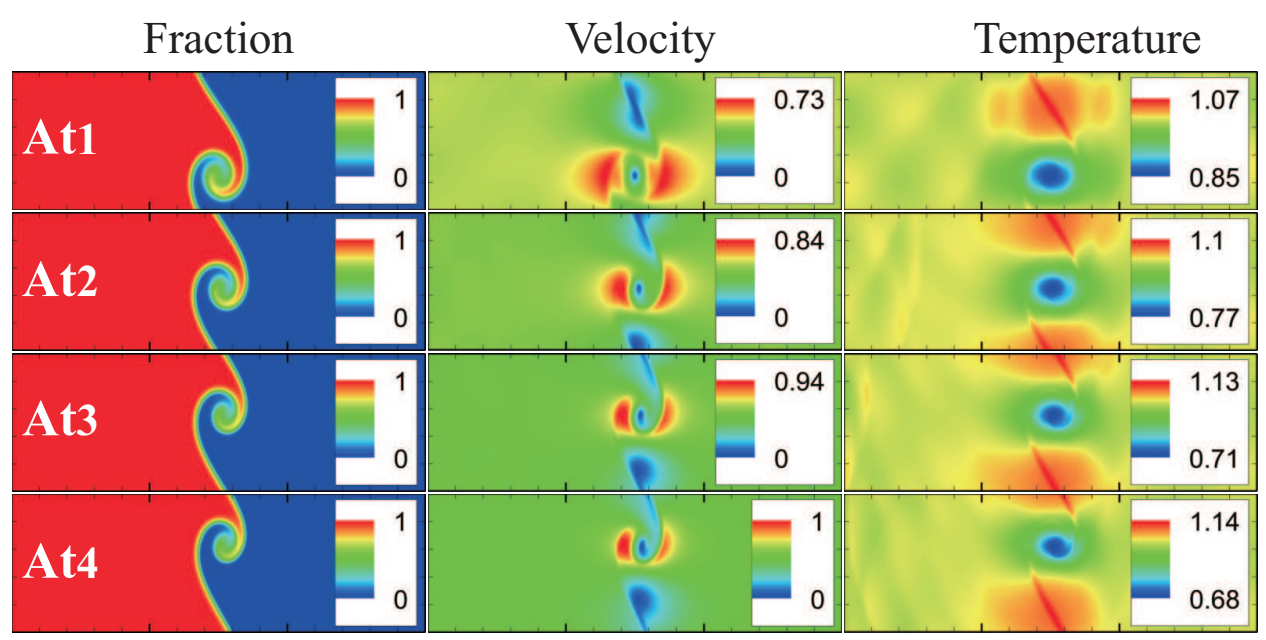

FIG. 12. Contours of physical quantities at time $t=1$. The leftmost, middle, and rightmost most columns are for the molar fraction of $A$, flow speed, and temperature, respectively. The four rows from top to bottom correspond to Atwood numbers $A t_{1}=0, A t_{2}=1 / 3, A t_{3}=1 / 2$, and $A t_{4}=3 / 5$, respectively.

and third columns that the minimum temperature is located at the vortex center, while the maximum temperature is located at the interface where the conversion from the shear kinetic energy to the internal energy is intense. In sum, the vortex has a smaller area, the light medium has faster speed, and the temperature has sharper gradients for a larger Atwood number.

Figuer 13 exhibits the evolution of nonequilibrium quantities with various Atwood numbers. Panel (a) shows that, for species $A$, the global strength of viscous stress, $\iint\left|\boldsymbol{\Delta}_{2}^{A *}\right| d x d y$, is smaller for a larger Atwood number. On the contrary, for species $B$, the global strength of the viscous stress, $\iint\left|\boldsymbol{\Delta}_{2}^{B *}\right| d x d y$, increases with the increasing Atwood number, as shown in panel (b). Physically, with the increasing mass ratio of species $A$ to $B$, the flow velocity of the heavy medium $A$ has a relatively small change, while the flow velocity of the light one $B$ has a relatively large change, during an interval. In addition, panel (c) shows that the global intensity of the heat flux, $\iint\left|\Delta_{3,1}^{A *}\right| d x d y$, decreases with the increasing Atwood number. While in panel (d), the peak of the global intensity of the heat flux, $\iint\left|\Delta_{3,1}^{B *}\right| d x d y$, is similarly high for different Atwood numbers. Because, mathematically, $\left|\boldsymbol{\Delta}_{3,1}^{A *}\right|$ is roughly inversely proportional to $m^{\sigma}$ [32]. Furthermore, for a larger Atwood number, the peaks of either $\iint\left|\boldsymbol{\Delta}_{2}^{\sigma *}\right| d x d y$ or $\iint\left|\boldsymbol{\Delta}_{3,1}^{\sigma *}\right| d x d y$ emerge later, because the amplitude of the perturbed 

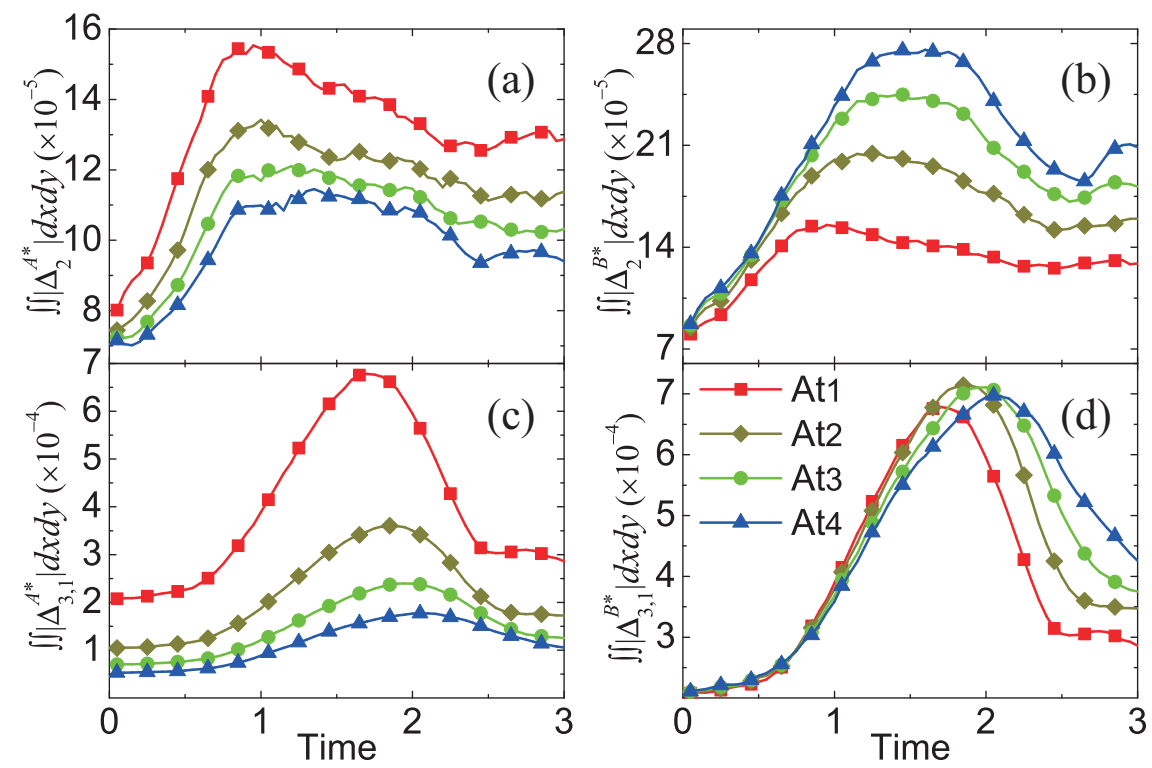

FIG. 13. Evolution of nonequilibrium quantities with various Atwood numbers: $A t_{1}=0$ (squares), $A t_{2}=1 / 3$ (diamonds), $A t_{3}=1 / 2$ (circles), and $A t_{4}=3 / 5$ (triangles), respectively. (a) $\iint\left|\boldsymbol{\Delta}_{2}^{A *}\right| d x d y$, (b) $\iint\left|\boldsymbol{\Delta}_{2}^{B *}\right| d x d y$, (c) $\iint\left|\boldsymbol{\Delta}_{3,1}^{A *}\right| d x d y$, (d) $\iint\left|\boldsymbol{\Delta}_{3,1}^{B *}\right| d x d y$.

interface grows more slowly [6].

Figure 14 illustrates the evolution of entropy of mixing, free enthalpy of mixing, and their growth rates with the Atwood number: $A t_{1}=0$ (squares), $A t_{2}=1 / 3$ (diamonds), $A t_{3}=1 / 2$ (circles), and $A t_{4}=3 / 5$ (triangles), respectively. It is evident in panel (a) that the entropy of mixing is lower for a larger Atwood number. Panel (b) shows that, for each Atwood number, the growth rate of entropy of mixing has three trends, i.e., firstly reducing, then increasing, and finally decreasing trends. In contrast, as shown in panels (c) and (d), the free enthalpy of mixing is higher for a larger Atwood number, and its growth rate shows firstly increasing, then reducing, and finally increasing trends. The trough (peak) in panel (b) and the peak (trough) in panel (d) take place almost simultaneously. Both the trough and peak emerge earlier for a smaller Atwood number in either panel (b) or (d). Because the growth rate of the perturbed interface is higher and the mixing area is wider for a smaller Atwood number, as shown in Fig. 12. 

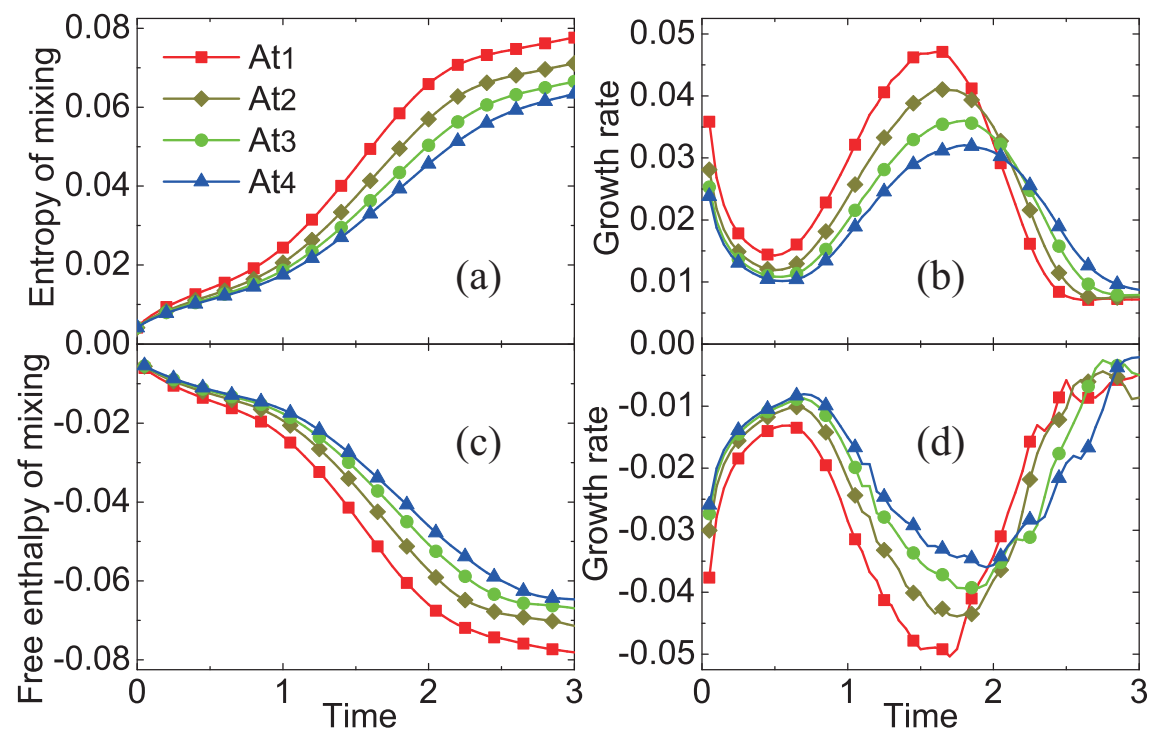

FIG. 14. (a) Entropy of mixing and (b) its growth rate, (c) free enthalpy of mixing and (d) its growth rate, with the Atwood number: $A t_{1}=0$ (squares), $A t_{2}=1 / 3$ (diamonds), $A t_{3}=1 / 2$ (circles), and $A t_{4}=3 / 5$ (triangles), respectively.

\section{CONCLUSIONS AND DISCUSSIONS}

As a mesoscopic kinetic method, the discrete Boltzmann method (DBM) not only recovers the traditional NS equations in the continuum limit, but also gives various nonequilibrium information. Besides, the discrete Boltzmann equation is in a uniform linear form, which is easy to code with high parallel efficiency. In this work, we adopt the DBM to investigate the nonequilibrium process of Kelvin-Helmholtz instability (KHI). First of all, two kinds of nonequilibrium manifestations, i.e., the viscous stresses and heat fluxes, are preliminarily studied. It is found that the global intensities of viscous stresses and heat fluxes become stronger for a larger relaxation time, and they firstly increase then decrease in the KHI process. Physically, the increasing nonequilibrium area enhances the GNEs, while the reducing physical gradients weaken the nonequilibrium effects.

In addition, the entropy of mixing is higher for a larger relaxation time, and its growth rate shows firstly reducing, then increasing, and finally decreasing trends in the KHI process. While the free enthalpy of mixing is lower for a larger relaxation time, and its growth rate has firstly increasing, then decreasing, and finally increasing trends. The free enthalpy of mixing is opposite to the entropy of mixing. Physically, there are competitive mechanisms. 
(i) The binary diffusion is fast when the gradient of molar fraction is sharp. The mixing speed reduces with the increasing width of the transition layer in the early stage. (ii) The mixing process is promoted by the increasing length of material interface in the second stage. (iii) As physical gradients are smoothed due to the binary diffusion, the mixing speed reduces and begins to approach zero in the final stage. It is of interest to find that the diffusion promotes the mixing process directly and initially, but hinders the mixing growth indirectly and eventually.

Moreover, we study the influence of Atwood number on the growth of the nonequilibrium KHI. With the increasing Atwood number, the global strength of viscous stress on the heavy medium reduces, while the one on the light medium increases. Physically, with the increasing mass ratio, the heavy medium has a relatively small velocity change, while the light has a relatively large velocity change, in the dynamic process. Since the heat flux is roughly inversely proportional to the molar mass, the global intensity of the heat flux reduces with the increasing molar mass. Furthermore, the growth rate of the perturbed interface increases with the increasing Atwood number. Consequently, for a larger Atwood number, the peaks of nonequilibrium manifestations emerge earlier, and the entropy of mixing and free enthalpy of mixing change faster.

\section{ACKNOWLEDGMENTS}

This work is supported by the Natural Science Foundation of China (under Grant Nos. 91441120, 51806116, 11875001, and 11602162), the China Postdoctoral Science Foundation under Grant No. 2017M620757, the Center for Combustion Energy at Tsinghua University, and the Natural Science Foundation of Hebei Province (A2017409014, ZD2017001 and A201500111). Support from the UK Engineering and Physical Sciences Research Council

under the project UK Consortium on Mesoscale Engineering Sciences (UKCOMES) (Grant Nos. EP/L00030X/1 and EP/R029598/1) is gratefully acknowledged.

[1] S. Chandrasekhar, Hydrodynamic and Hydromagnetic Stability (Oxford University Press, New York, 1961). 
[2] G. K. Batchelor, An introduction to fluid dynamics (Cambridge university press, Cambridge, 2000).

[3] H. Hasegawa, M. Fujimoto, T. D. Phan, H. Reme, A. Balogh, M. Dunlop, C. Hashimoto, and R. TanDokoro, Nature 430, 755 (2004).

[4] S. Som and S. Aggarwal, Combust. Flame 157, 1179 (2010).

[5] H. Yang, Z. Xu, E. K. Lim, S. Kim, K. S. Cho, Y. H. Kim, J. Chae, K. Cho, and K. Ji, Astrophys. J. 857 (2018).

[6] L. Wang, W. Ye, X. He, J. Wu, Z. Fan, C. Xue, H. Guo, W. Miao, Y. Yuan, J. Dong, G. Jia, J. Zhang, Y. Li, J. Liu, M. Wang, Y. Ding, and W. Zhang, Sci. China Phys. Mech. Astron. 60 (2017).

[7] B. Akula, P. Suchandra, M. Mikhaeil, and D. Ranjan, J. Fluid Mech. 816, 619 (2017).

[8] M. Brouillette, Annu. Rev. Fluid Mech. 34, 445 (2002).

[9] Y. Zhou, Phys. Rep. 723, 1 (2017).

[10] W. C. Wan, G. Malamud, A. Shimony, C. A. Di Stefano, M. R. Trantham, S. R. Klein, D. Shvarts, C. C. Kuranz, and R. P. Drake, Phys. Rev. Lett. 115 (2015).

[11] Y. Liu, P. Tan, and L. Xu, Proc. Natl. Acad. Sci. U.S.A. 112, 3280 (2015).

[12] H. G. Lee and J. Kim, Eur. J. Mech. B. Fluids 49, 77 (2015).

[13] Y. Li, X. Geng, Z. Liu, H. Wang, and D. Zang, Fluid Dyn. Res. 50, 045512 (2018).

[14] J. Ashwin and R. Ganesh, Phys. Rev. Lett. 104 (2010).

[15] O. Tumuklu, D. A. Levin, and V. Theofilis, Phys. Fluids 30 (2018).

[16] Q. Li, K. H. Luo, Q. J. Kang, Y. L. He, Q. Chen, and Q. Liu, Prog. Energy Combust. Sci. $\mathbf{5 2 ,} 62(2016)$.

[17] L. Wang, A. Teng, W. Ye, Z. Fan, Y. Tao, C. Lin, and Y. Li, Acta Phys. Sin. 58, 8426 (2009).

[18] S. Succi, The Lattice Boltzmann Equation for Fluid Dynamics and Beyond (Oxford University Press, New York, 2001).

[19] J. Meng, Y. Zhang, N. G. Hadjiconstantinou, G. A. Radtke, and X. Shan, J. Fluid Mech. 718, 347 (2013).

[20] C. Zhuo and C. Zhong, Int. J. Comput. Fluid D. 30, 543 (2016).

[21] R. Y. Zhang, X. Y. He, G. Doolen, and S. Y. Chen, Adv. Water Resour. 24, 461 (2001).

[22] H. G. Lee and J. Kim, Eur. J. Mech. B. Fluids 49, 77 (2015).

[23] H. Liang, B. C. Shi, and Z. H. Chai, Phys. Rev. E 93, 1 (2016). 
[24] H. Amirshaghaghi, M. Rahimian, and H. Safari, Int. Commun. Heat Mass Transfer 75, 282 (2016).

[25] A. Fakhari, M. Geier, and T. Lee, J. Comput. Phys. 315, 434 (2016).

[26] R. C. V. Coelho, M. Mendoza, M. M. Doria, and H. J. Herrmann, Phys. Rev. B 96, 184307 (2017).

[27] Y. Gan, A. Xu, G. Zhang, and Y. Li, Phys. Rev. E 83 (2011).

[28] F. Chen, A. Xu, and G. Zhang, Front. Phys. 11, 114703 (2016).

[29] H. Lai, A. Xu, G. Zhang, Y. Gan, Y. Ying, and S. Succi, Phys. Rev. E 94, 023106 (2016).

[30] C. Lin, A. Xu, G. Zhang, Y. Li, and S. Succi, Phys. Rev. E 89 (2014).

[31] C. Lin, A. Xu, G. Zhang, and Y. Li, Combust. Flame 164, 137 (2016).

[32] C. Lin, K. H. Luo, L. Fei, and S. Succi, Sci. Rep. 7 (2017).

[33] V. Sofonea and R. F. Sekerka, Physica A 299, 494 (2001).

[34] H. Zhang and F. Zhuang, Adv. Appl. Mech. 29, 193 (1991).

[35] M. Watari and M. Tsutahara, Phys. Rev. E 67 (2003).

[36] C. Lin, K. H. Luo, Y. Gan, and H. Lai, Commun. Theor. Phys. 69, 722 (2018). 